\title{
Dependence of the flux-creep activation energy on current density and magnetic field for a $\mathrm{Ca}_{10}\left(\mathrm{Pt}_{3} \mathrm{As}_{8}\right)\left[\left(\mathrm{Fe}_{1-x} \mathrm{Pt}_{x}\right)_{2} \mathrm{As}_{2}\right]_{5}$ single crystal
}

\author{
J. Ge, ${ }^{1}$ J. Gutierrez, ${ }^{1}$ J. Li ${ }^{1,2}$ J. Yuan, ${ }^{3,2}$ H.-B. Wang, ${ }^{2,4}$ K. Yamaura, ${ }^{2,5}$ \\ E. Takayama-Muromachi, ${ }^{5,6}$ and V. V. Moshchalkov ${ }^{1}$ \\ ${ }^{1}$ INPAC-Institute for Nanoscale Physics and Chemistry, KU Leuven, Celestijnenlaan 200D, B-3001 Leuven, \\ Belgium \\ ${ }^{2}$ Superconducting Properties Unit, National Institute for Materials Science, 1-1 Namiki, Tsukuba, \\ Ibaraki 305-0044, Japan \\ ${ }^{3}$ Condensed Matter Physics, Institute of Physics, Chinese Academy of Sciences, Beijing 100190, China \\ ${ }^{4}$ Research Institute of Superconductor Electronics, Nanjing University, Nanjing 210093, China \\ ${ }^{5}$ Graduate School of Chemical Sciences and Engineering, Hokkaido University, Sapporo, Hokkaido 060-0810, \\ Japan \\ ${ }^{6}$ International Center for Materials Nanoarchitectonics (WPI-MANA), National Institute for Materials \\ Science, 1-1 Namiki, Tsukuba, Ibaraki 305-0044, Japan
}

(Received 13 February 2014; accepted 6 March 2014; published online 17 March 2014)

\begin{abstract}
We have performed detailed ac susceptibility measurements to investigate the vortex dynamics in a $\mathrm{Ca}_{10}\left(\mathrm{Pt}_{3} \mathrm{As}_{8}\right)\left[\left(\mathrm{Fe}_{1-x} \mathrm{Pt}_{x}\right)_{2} \mathrm{As}_{2}\right]_{5}$ single crystal as a function of temperature, frequency, ac amplitude, and $\mathrm{dc}$ field. The field dependence of the activation energy $U$ is derived in the framework of thermally activated flux creep theory, yielding a power law dependence of $U \sim H^{\alpha}$ with $\alpha \approx-1.0$ for $\mathrm{H}$ above $0.30 \mathrm{~T}$, while below $0.3 \mathrm{~T} U$ is independent of the field. The activation energy reaches $10^{4} \mathrm{~K}$ at low fields, suggesting strong pinning in the material. The nonlinear function of the activation energy vs. the current density is determined, which shows logarithmic dependence $U(J) \propto \operatorname{lnJ}$. (C) 2014 AIP Publishing LLC. [http://dx.doi.org/10.1063/1.4868871]
\end{abstract}

Ever since the discovery of superconductivity in $\mathrm{LaFeAsO}_{0.9} \mathrm{~F}_{0.1},{ }^{1}$ intensive efforts have been focused on searching for new series of superconductors in related systems. Very recently, the successful synthesis of $\mathrm{Ca}_{10}\left(\mathrm{Pt}_{n} \mathrm{As}_{8}\right)\left[\left(\mathrm{Fe}_{1-x} \mathrm{Pt}_{x}\right)_{2} \mathrm{As}_{2}\right]_{5}$ with $\mathrm{n}=3$ (10-3-8 phase) and $\mathrm{n}=4$ (10-4-8 phase) has attracted a lot of interest. ${ }^{2-9}$ Mostly, this system serves as a model of the impact on the superconducting properties by intermediary layers, which is yet an open question. The critical temperature $T_{c}$ up to $13 \mathrm{~K}$ and $38 \mathrm{~K}$ has been reported in $10-3-8$ phase and 10-4-8 phase, respectively. The new superconducting system shares similar properties with other iron-based superconductors, such as very high upper critical fields, ${ }^{10}$ high critical current, ${ }^{11}$ and multiband nature. ${ }^{12}$ Besides the similarities, the Ca-Fe-Pt-As system also exhibits many unique properties which resemble those of the high $T_{c}$ cuprates. For example, very high anisotropy $\gamma \equiv H_{c 2}^{a b} / H_{c 2}^{c} \sim 10$ has been observed close to $T_{c}{ }^{13}$ and very large penetration depth $(\sim 1000 \mathrm{~nm})$, indicating small superfluid density, have been observed in the 10-3-8 phase. The Ca-Fe-Pt-As system has a layered $\mathrm{Ca}-\left(\mathrm{Pt}_{n} \mathrm{As}_{8}\right)-$ $\mathrm{Ca}-\left(\mathrm{Fe}_{2} \mathrm{As}_{2}\right)$ stack, which is analogous to the structure in $\mathrm{Bi}_{2} \mathrm{Sr}_{2} \mathrm{Ca}_{n-1} \mathrm{Cu}_{n} \mathrm{O}_{2 n+4+x}$ (BSCCO, $\mathrm{n}=1-3$ ) systems. ${ }^{14}$ In the latter case, the high $T_{c}$ is suggested to arise from the enhanced $\mathrm{CuO}_{2}$ plane coupling through the intermediary layers. ${ }^{15-18}$ Therefore, the Ca-Fe-Pt-As system is an excellent candidate to provide clues to the understanding of high $T_{c}$ superconductivity that also determines parameters such as high upper critical fields and complex vortex dynamics due to thermal fluctuations.

Besides the critical temperature, another important parameter for high $T_{c}$ superconductors is the critical current density $J_{c}$, i.e., the ability of carrying current without dissipation due to vortex motion. Normally, it is difficult to measure
$J_{c}$ directly in a bulk sample, especially at very low temperatures. Note that $J_{c}$ of iron-based superconductors reaches values as high as $10^{7} \mathrm{~A} / \mathrm{cm}^{2}{ }^{19}$ However, $J_{c}$ is determined by the pinning properties of the sample. Dissipation occurs only above the activation energy $U$ when the vortices overcome the attractive force exerted by the pinning centers. Therefore, the study of the activation energy in the Ca-Fe-Pt-As system and its comparison to other iron-based superconductors as well as high $T_{c}$ cuprates is important for the understanding of vortex dynamics in high $T_{c}$ superconductors.

Among various ways to study the activation energy, the ac susceptibility has the advantages of high sensitivity, fast reaction to vortex dynamics, and convenience of handling. ${ }^{20-24} \mathrm{In}$ this paper, we have used the ac susceptibility measurements to study the vortex dynamics in a $\mathrm{Ca}_{10}\left(\mathrm{Pt}_{3} \mathrm{As}_{8}\right)\left[\left(\mathrm{Fe}_{1-x} \mathrm{Pt}_{x}\right)_{2} \mathrm{As}_{2}\right]_{5}$ single crystal at different ac fields, frequencies, and dc fields. The activation energy as a function of temperature, magnetic field, and current density has been determined.

The studied single crystal $\mathrm{Ca}_{10}\left(\mathrm{Pt}_{3} \mathrm{As}_{8}\right)\left[\left(\mathrm{Fe}_{1-x} \mathrm{Pt}_{x}\right)_{2} \mathrm{As}_{2}\right]_{5}$ was prepared using a high-pressure technique as reported in Ref. 25. Inset of Fig. 1(a) displays an optical image of a single crystal. The mirror-like surface of the crystal was characterized by x-ray diffraction (XRD) in a Rigaku Ultima-IV diffractometer using $\mathrm{CuK} \alpha$ radiation. The result is shown in Fig. 1(a). An obvious orientation toward [00n] is observed, indicating that the c-axis is perpendicular to the single crystal plane. Single crystals were also grounded and studied by a powder XRD method, and the results yield the tetragonal SrZnSb2-type structure $(\mathrm{P} 4 / \mathrm{n})$ as reported elsewhere. ${ }^{3}$ The sample, with a size of $\sim 0.8 \times 1.3 \times 0.5 \mathrm{~mm}^{3}$, is characterized by $T_{c}=9.7 \mathrm{~K}$, $\Delta T_{c}=1.0 \mathrm{~K}$ (10\%-90\% criterion). The ac susceptibility measurements were performed using a Quantum-Design Physical Property Measurement System (PPMS) with the external dc 

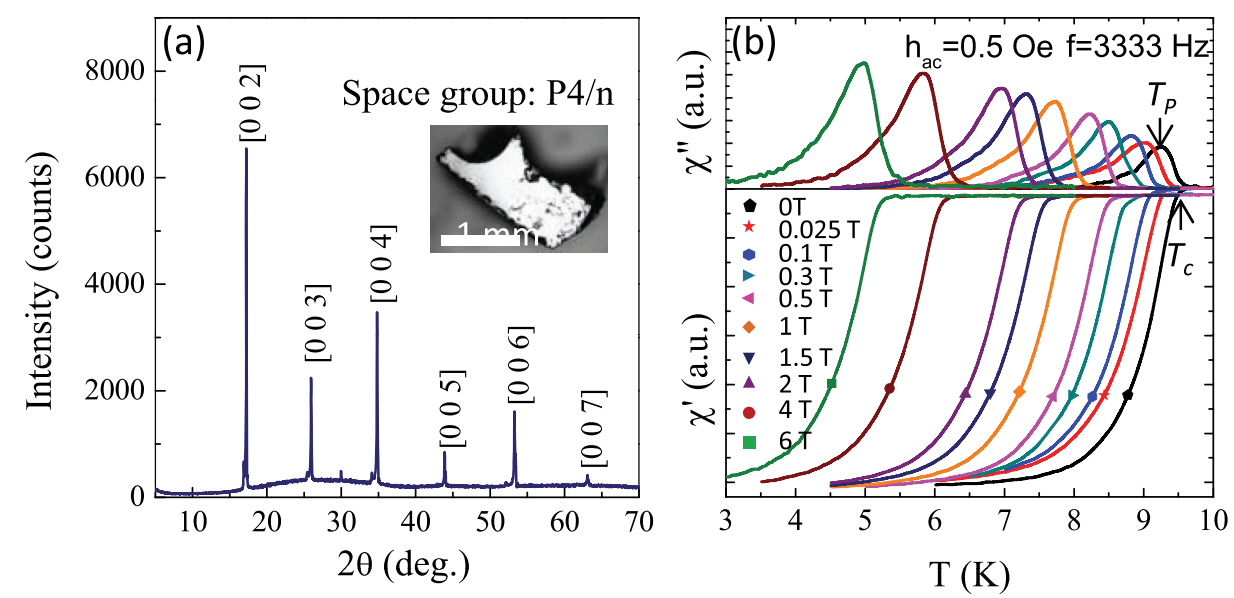

FIG. 1. (a) XRD pattern of the measured single crystal. The inset shows an optical microscope image of a $\mathrm{Ca}_{10}\left(\mathrm{Pt}_{3} \mathrm{As}_{8}\right)\left[\left(\mathrm{Fe}_{1-x} \mathrm{Pt}_{x}\right)_{2} \mathrm{As}_{2}\right]_{5}$ crystal. (b) Temperature dependence of outof-phase and in-phase of the ac susceptibility under various magnetic fields for $h_{a c}=1$ Oe at $f=3333 \mathrm{~Hz}$. field up to $6 \mathrm{~T}$, an ac field with the amplitude and frequency in the range of $h_{a c}=0.5-12 \mathrm{Oe}$ and $133-9777 \mathrm{~Hz}$, respectively. Both dc and ac fields are perpendicular to the sample surface (ab-plane) in all the measurements.

The ac susceptibility has been used to study the vortex dynamics in other iron-based superconductors, e.g., 1111family $^{22}$ and 122-family. ${ }^{24,26}$ It is known that the in-phase ac susceptibility exhibits a sharp decrease at $T_{c}$ while at lower temperatures the out-of-phase shows a dissipation peak at $T_{p}$ due to the melting of the vortex lattice. Therefore, at low ac amplitude and frequency, $T_{p}$ can be used as a rough estimation of $T_{\text {irr }}$.

Fig. 1(b) shows the temperature dependence of ac susceptibility under various dc fields. As dc field increases, the transition temperature shifts to lower temperatures while the transition width remains almost constant. The relatively small $\delta T_{c}$ and its almost $H$-independent behavior suggest the high quality of our sample. To study the activation energy $U$, the thermally activated flux creep model is used ${ }^{27}$

$$
U\left(T_{p}, H_{d c}, J\right)=U\left(T_{p}\right) U\left(H_{d c}, J\right)=T_{p} \ln \frac{1}{f t_{0}},
$$

where $U\left(T_{p}\right)$ accounts for the temperature dependence of $U$, $t_{0}=\pi T_{p} d^{2} /\left(2 H c v_{0}|\partial U|\right)$ is the time scale, $2 d$ is the thickness of the slab sample, $v=v_{0} e^{-U / T}$ is the velocity of the flux diffusion.

Numerical simulations have shown that, during the penetration of the ac field into a superconductor, the static critical state model (Bean model) can be used. ${ }^{28}$ The magnetic field profile inside the sample can be regarded as a straight line. At the peak temperature $T_{p}$, where the flux front reaches the center of the sample, the current density can be estimated using

$$
J=h_{a c} / d
$$

It is clear from Eq. (1) that, at a constant $h_{a c}$ (constant current density) and $H_{d c}$, a plot of $\ln f$ versus $U\left(T_{p}\right) / T_{p}$ should give a straight line with the slope of $U\left(J, H_{d c}\right)$. By varying $H_{d c}$ while keeping $h_{a c}$ constant, one can get the field dependence of the activation energy and vice versa.

Fig. 2(a) presents some typical curves of the temperature dependence of ac susceptibility under various frequencies at $H_{d c}=6 \mathrm{~T}$ and $h_{a c}=0.5 \mathrm{Oe}$. As the frequency decreases, the peak transition shifts to lower temperatures. This is reasonable considering that the vortices have more time for relaxation at lower frequency, resulting in full penetration at lower temperatures. Similar behavior has also been observed in other superconducting systems which are explained in terms of flux creep. $^{27,29}$ The effect of ac amplitude on the flux dynamics at each frequency is also measured. Fig. 2(b) presents some typical curves of the temperature dependence of ac susceptibility in the ac field range of $0.5-12 \mathrm{Oe}$ with $H_{d c}=6 \mathrm{~T}$ and $f=9777 \mathrm{~Hz}$. With increasing $h_{a c}$, the low temperature part of $\chi^{\prime}$ shifts downward corresponding to an increase in the screening current. At the same time, the $\chi^{\prime \prime}$ peak becomes broader, shifts to lower temperature, and its height increases. All these features support a scenario of nonlinear response of the sample which is well predicted by the critical state model. ${ }^{30}$
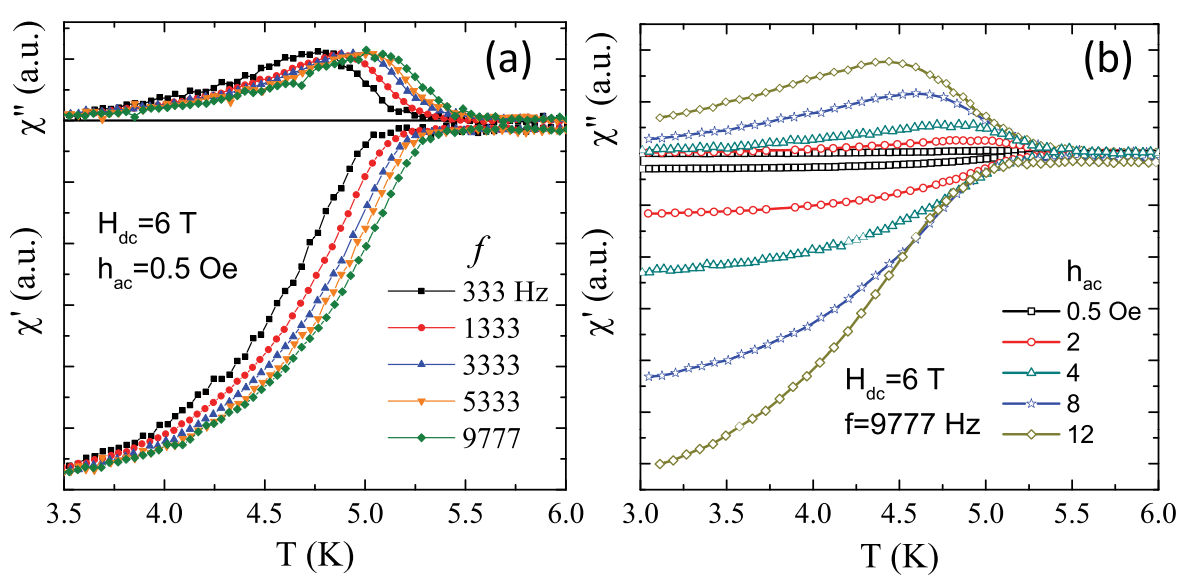

FIG. 2. (a) Temperature dependence of out-of-phase and in-phase of the ac susceptibility at $H_{d c}=6 \mathrm{~T}, h_{a c}=0.5 \mathrm{Oe}$ and $f$ varying from $333 \mathrm{~Hz}$ to $9777 \mathrm{~Hz}$. (b) Temperature dependence of out-ofphase and in-phase of the ac susceptibility at $H_{d c}=6 \mathrm{~T}, f=9777 \mathrm{~Hz}$, and $h_{a c}$ varying from $0.5 \mathrm{Oe}$ to $12 \mathrm{Oe}$. 
In our measurements, the maximum variation of the $T_{p}$ as a function of frequency is around $1 \mathrm{~K}$. Therefore, the activation energy cannot be regarded as an isothermal parameter, the explicit temperature dependence must be taken into account. ${ }^{26}$ According to Ref. 31, $U(T)$ has the following form:

$$
U(T) \equiv\left[1-\left(T / T_{c}\right)^{r}\right]^{n},
$$

where $\mathrm{r}$ and $\mathrm{n}$ are fitting parameters to make the $U\left(T_{p}\right) / T_{p}$ vs. $\ln f$ a straight line at each field. Here, by adjusting $\mathrm{r}=2$ and $\mathrm{n}=2$, we have found that the Arrhenius law can be established. Such index values have also been used to account for the $U(T)$ in $\mathrm{HgBa}_{2} \mathrm{Ca}_{2} \mathrm{Cu}_{3} \mathrm{O}_{x}$ (Ref. 32) and $\mathrm{MgB}_{2}$ (Ref. 20) materials.

Fig. 3(a) shows the semi-log plot of $f$ vs. $U\left(T_{p}\right) / T_{p}$ at various dc fields for $\mathrm{H} \| \mathrm{c}$. For clarity, only the data measured at $H=0.05,0.5,2,4$, and $6 \mathrm{~T}$ are shown. The straight lines are linear fitting to the data as discussed above. As mentioned above, the irreversibility line (IL) can be estimated from $T_{p}$ vs. H at extremely low frequency (corresponding to low voltage criterion). Here, by extrapolating the linear fit in Fig. 3(a) to $\ln f=0(f=1 \mathrm{~Hz})$, the IL can be inferred. The IL together with the upper critical fields derived from the onset diamagnetic signal in $\chi^{\prime}$ are shown in the inset of Fig. 3(a). The dashed line is the fitting using the empirical formula: $H_{i r r}(T)=H_{0}\left(1-T_{i r r} / T_{c}\right)^{\beta} \quad$ with $\quad H_{0}=22.46 \mathrm{~T} \quad$ and $\beta=2.16 . \beta=2$ is the expected value for the lattice melting phase boundary close to $T_{c}$, ${ }^{33}$ which is consistent with the values found for the 1111-family ( $\beta=1.5$ ), ${ }^{22,23} 122$-family ( $\beta=1.52$ ) iron-based superconductors, ${ }^{34}$ and $\mathrm{TlSr}_{2} \mathrm{Ca}_{2} \mathrm{Cu}_{3} \mathrm{O}_{\mathrm{y}}$ $(\beta=1.5){ }^{35}$ In these systems, $\beta=1.5$ suggests vortex liquid-glass transition due to the presence of disorder. ${ }^{36}$ It has been reported that the Ginzburg number $G i=0.16$ for the 10-3-8 phase, ${ }^{6}$ which is bigger than those of the cuprates $\mathrm{YBa}_{2} \mathrm{Cu}_{3} \mathrm{O}_{7}(G i=0.01)$ and $\mathrm{BSCCO}(G i=0.1)$. Therefore, large thermal fluctuations in the material are expected. However, our measurements show that the IL is very close to the $H_{c 2}$ line. We find the ratio $H_{i r r}(T) / H_{c 2}(T) \sim 0.65$ for $T<6 \mathrm{~K}$ in the 10-3-8 phase. This ratio is higher than those found in the cuprates, and it is comparable to those of low- $T_{c}$ superconductors such as $\mathrm{Nb}-\mathrm{Ti}$ and $\mathrm{NbSn}_{3}$ (where $H_{\text {irr }}(T) / H_{c 2}(T) \sim 0.85$ (Ref. 37)). This last comparison indicates something surprising, since the 10-3-8 phase has a much higher Gi number as compared to the low- $T_{c}$ superconductors. In this case, one should expect large thermal fluctuations, yet the irreversibility line lies very close to the $H_{c 2}(T)$ line like in low- $T_{c}$ superconductors.

The field dependence of the activation energy $U(H)$, representing the effective pinning barrier, is shown in Fig. 3(b). The pinning potential value reaches $10^{4} \mathrm{~K}$ at low fields, comparable with the reported values of most iron-based superconductors, like $\mathrm{SmFeAsO}_{0.8} \mathrm{~F}_{0.2} \quad\left(\sim 5 \times 10^{4} \mathrm{~K}\right),{ }^{22}$ $\mathrm{Ba}_{0.72} \mathrm{~K}_{0.28} \mathrm{Fe}_{2} \mathrm{As}_{2}\left(\sim 10^{4} \mathrm{~K}\right){ }^{38} \mathrm{~K}_{0.8} \mathrm{Fe}_{2} \mathrm{Se}_{2}\left(\sim 6 \times 10^{5} \mathrm{~K}\right),{ }^{26}$ and $\mathrm{FeTe}_{0.5} \mathrm{Se}_{0.5}\left(\sim 4.6 \times 10^{3} \mathrm{~K}\right) .{ }^{39}$ This implies strong pinning in the material which is promising for their potential applications. Moreover, the field dependence of $U(H)$ displays two regimes well separated by a crossover field $H_{c r}=$ $0.30 \mathrm{~T}$. Below $H_{c r}, U(H)$ is independent of the magnetic field, while above $H_{c r}, U(H)$ follows the dependence of $H^{-1.0}$. Such a crossover has also been observed in other superconducting systems where the weak field dependence of the activation energy at low fields is attributed to single vortex pinning. ${ }^{22,40}$ Similarly, an $\sim H^{-1}$ dependence has been previously derived using the Anderson-Kim model of the activation energy combined with the Ginzburg-Landau expressions for the coherence length, thermodynamic critical field, and depairing critical current density. ${ }^{41,42}$ Note that similar field dependence has also been observed in other superconducting systems, such as iron-based superconductors, ${ }^{22,26} \mathrm{La}_{1.86} \mathrm{Sr}_{0.14} \mathrm{CuO}_{4}$ (Ref. 31) and $\mathrm{MgB}_{2} .{ }^{20}$

It follows from Eq. (2) that the current density at $T_{p}$ is proportional to the applied ac amplitude $h_{a c}$. To study the relationship between current density and the activation energy, ac susceptibility measurements have been performed under various frequencies and ac fields as indicated in Fig. 4. Fig. 4(a) displays the $U\left(T_{p}\right) / T_{p}$ as a function of frequency in a semi-log plot at $H_{d c}=0.1 \mathrm{~T}$ and various ac fields. Similar plot has also been made at high field regime $(H=6 \mathrm{~T})$, as shown in Fig. 4(c). The experimental data can be fitted very well by straight lines. The activation energy at each current density can be then derived from the slopes of the straight lines, which is shown in Figs. 4(b) and 4(d). It is clear that $U(J)$ follows a logarithmic dependence- $\ln J$ with current density. Such behavior has been observed before in different height $-T_{c}$ superconductors. ${ }^{43-46}$ It has been proposed that the physical mechanism yielding such dependence arises due to an enhanced hopping rate of vortex bundles, which may become orders of magnitude larger than the random thermal equilibrium motion leading to a collective creep of vortices. ${ }^{44,47}$ This is a dissipation mechanism occurring as a transition between a classical creep at
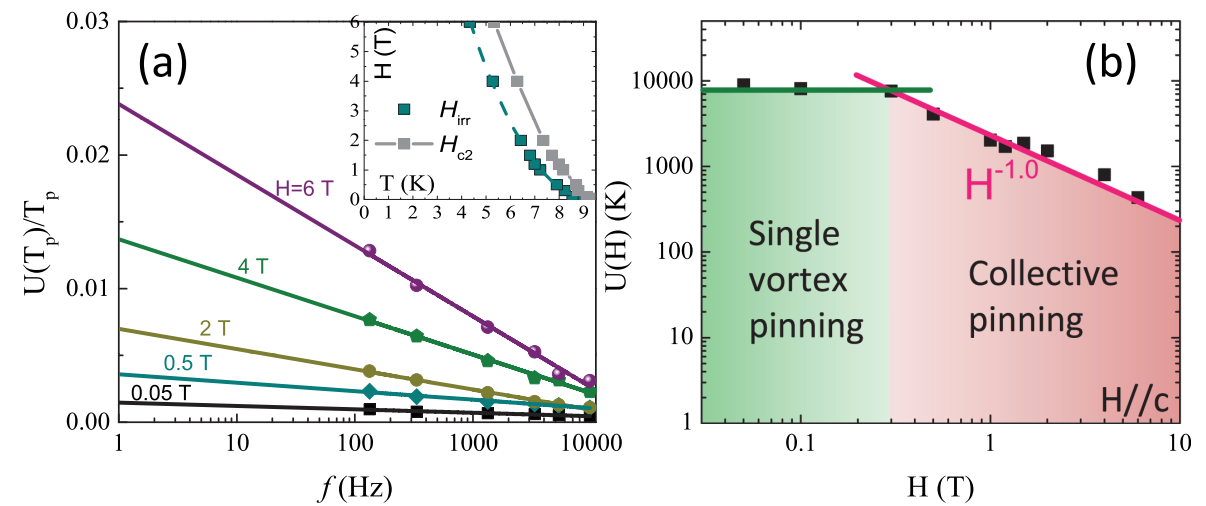

FIG. 3. (a) Frequency dependence of $U(T) / T_{p}$ measured at various magnetic fields as indicated in the panel. The inset presents the vortex phase diagram. The dashed line is a fitting using the empirical formula $H_{\text {irr }}(T)=22.46$ $\times\left(1-T_{i r r} / T_{c}\right)^{2.16}$. The straight lines are linear fittings to the data. (b) The activation energy as a function of field in log-log scale. The solid line above $H>0.3 \mathrm{~T}$ is fit to the data using the relation $U=H^{-\alpha}$ with $\alpha=1.0$. 


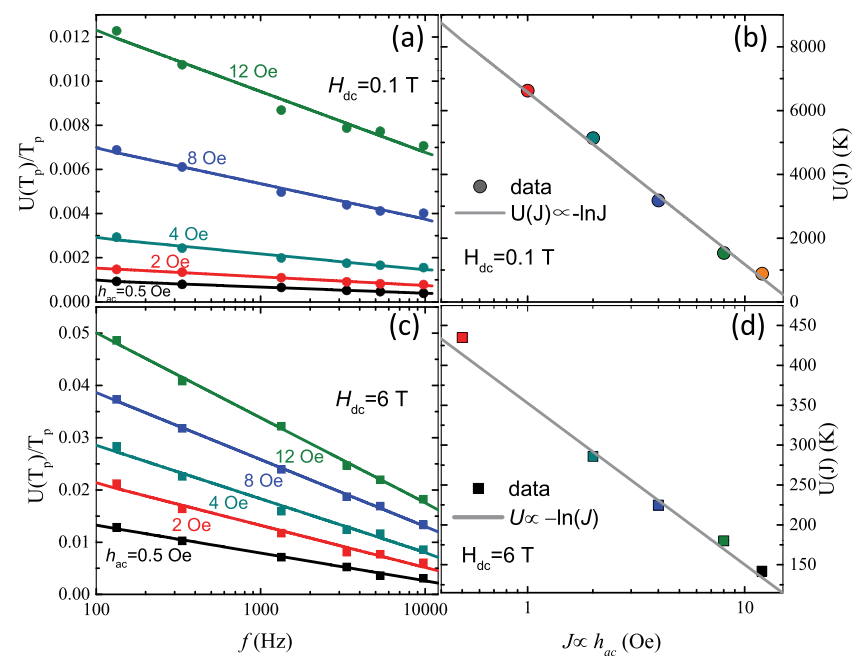

FIG. 4. (a) and (c) Frequency dependence of $U\left(T_{p}\right) / T_{p}$ measured at various ac fields (current densities) as indicated in the panel under magnetic dc field of $0.1 \mathrm{~T}$ and $6 \mathrm{~T}$, respectively. Solid lines are linear fits to the data. Activation energy as a function of the current density at (b) $H_{d c}=0.1 \mathrm{~T}$ and (d) $H_{d c}=6 \mathrm{~T}$. The solid lines are the fitting curves with the relation $U(J) \propto-\ln J$.

even lower $J$ (which leads to a $U \sim J^{-\mu}$ ) and flux flow dissipation at high $\mathrm{J}$.

In summary, detailed ac susceptibility measurements have been performed to study the flux dynamics in a $\mathrm{Ca}_{10}\left(\mathrm{Pt}_{3} \mathrm{As}_{8}\right)\left[\left(\mathrm{Fe}_{1-x} \mathrm{Pt}_{x}\right)_{2} \mathrm{As}_{2}\right]_{5}$ single crystal. With the temperature dependence of the activation energy $U(T)$ $=\left[1-\left(T / T_{c}\right)^{2}\right]^{2}$, the pinning barrier energy as a function of magnetic field and current density is determined. The activation energy reaches $10^{4} \mathrm{~K}$ at low fields and is field independent up to $H_{c r}=0.3 \mathrm{~T}$, indicating very strong pinning. Above $H_{c r}$, the activation energy decays with the magnetic field as $U \sim H^{-1}$ which is typical for collective pinning. The observed nonlinear current dependence at low current densities arises from the collective creep of vortices, as have been previously observed in high- $T_{c}$ superconductors. The strong pinning observed here and in other FeAs-based superconductors $\left(10^{3}-10^{5} \mathrm{~K}\right)$, compared with cuprates crystals $\left(\mathrm{LSCO} \sim 10^{3} \mathrm{~K}^{48}{ }^{48} \mathrm{YBCO} \sim 10^{3} \mathrm{~K}^{49}\right.$ Bi2212 $\sim 10^{3}$ (Ref. $50)$ ), suggests that it might be an intrinsic property of the iron-based superconductors, probably arises from the unique layered structure and small coherence length of the materials combined with their small anisotropy.

The work at KU Leuven was supported by the FWO, the Methusalem Funding by the Flemish Government and the MP1201 COST Action. The work at Japan is financially supported by the World Premier International Research Center from MEXT, the Grants-in-Aid for Scientific Research from JSPS Japan, the Funding Program for World-Leading Innovative $R \& D$ on Science and Technology (FIRST Program) from JSPS, the Advanced Low Carbon Technology Research and Development Program (ALCA) from JST, and the National Natural Science Foundation of China (No. 11234006).

${ }^{1}$ Y. Kamihara, T. Watanabe, M. Hirano, and H. Hosono, J. Am. Chem. Soc. 130, 3296 (2008).

${ }^{2}$ M. Nohara, S. Kakiya, and K. Kudo, in International Workshop on Novel Superconductors and Super Materials, Tokyo, Japan, 6-8 March, 2011.
${ }^{3}$ N. Ni, J. M. Allred, B. C. Chan, and R. J. Cava, Proc. Natl. Acad. Sci. U.S.A. 108, E1019 (2011).

${ }^{4}$ S. Kakiya, K. Kudo, Y. Nishikubo, K. Oku, E. Nishibori, H. Sawa, T. Yamamoto, T. Nozaka, and M. Nohara, J. Phys. Soc. Jpn. 80, 093704 (2011).

${ }^{5}$ M. Neupane, C. Liu, S. Y. Xu, Y. J. Wang, N. Ni, J. M. Allred, L. A. Wray, N. Alidoust, H. Lin, R. S. Markiewicz, A. Bansil, R. J. Cava, and M. Z. Hasan, Phys. Rev. B 85, 094510 (2012).

${ }^{6}$ J. Kim, F. Ronning, N. Haberkorn, L. Civale, E. Nazaretski, N. Ni, R. J. Cava, J. D. Thompson, and R. Movshovich, Phys. Rev. B 85, 180504(R) (2012).

${ }^{7}$ S. Thirupathaiah, T. Sturzer, V. B. Zabolotnyy, D. Johrendt, B. Buchner, and S. V. Borisenko, Phys. Rev. B 88, 140505(R) (2013).

${ }^{8}$ Z. J. Xiang, X. G. Luo, J. J. Ying, X. F. Wang, Y. J. Yan, A. F. Wang, P. Cheng, G. J. Ye, and X. H. Chen, Phys. Rev. B 85, 224527 (2012).

${ }^{9}$ T. Sturzer, G. Derondeau, and D. Johrendt, Phys. Rev. B 86, 060516(R) (2012).

${ }^{10}$ E. Mun, N. Ni, J. M. Allred, R. J. Cava, O. Ayala, R. D. Mcdonald, N. Harrison, and V. S. Zapf, Phys. Rev. B 85, 100502 (2012).

${ }^{11}$ Q. P. Ding, Y. Tsuchiya, S. Mohan, T. Taen, Y. Nakajima, and T. Tamegai, Phys. Rev. B 85, 104512 (2012).

${ }^{12}$ K. Cho, M. A. Tanatar, H. Kim, W. E. Straszheim, N. Ni, R. J. Cava, and R. Prozorov, Phys. Rev. B 85, 020504(R) (2012).

${ }^{13}$ C. Lohnert, T. Sturzer, M. Tegel, R. Frankovsky, G. Friederichs, and D. Johrendt, Angew. Chem., Int. Ed. 50, 9195 (2011).

${ }^{14}$ A. Damascelli, Z. Hussain, and Z.-X. Shen, Rev. Mod. Phys. 75, 473 (2003).

${ }^{15}$ P. A. Sterne and C. S. Wang, J. Phys. C: Solid State Phys. 21, L949 (1988).

${ }^{16}$ Z. Z. Sheng, A. M. Hermann, A. El Ali, C. Almasan, J. Estrada, T. Datta, and R. J. Matson, Phys. Rev. Lett. 60, 937 (1988).

${ }^{17}$ S. S. P. Parkin, V. Y. Lee, E. M. Engler, A. I. Nazzal, T. C. Huang, G. Gorman, R. Savoy, and R. Beyers, Phys. Rev. Lett. 60, 2539 (1988).

${ }^{18}$ L. Gao, Z. J. Huang, R. L. Meng, J. G. Lin, F. Chen, L. Beauvais, Y. Y. Sun, Y. Y. Xue, and C. W. Chu, Physica C 213, 261 (1993).

${ }^{19}$ J. Li, J. Yuan, Y. H. Yuan, J. Ge, M. Y. Li, H. L. Feng, P. J. Pereira, A. Ishii, T. Hatano, A. V. Silhanek et al., Appl. Phys. Lett. 103, 062603 (2013).

${ }^{20}$ M. J. Qin, X. L. Wang, S. Soltanian, A. H. Li, H. K. Liu, and S. X. Dou, Phys. Rev. B 64, 060505(R) (2001).

${ }^{21}$ S. Y. Ding, C. Ren, H. J. Yi, Z. Y. Zeng, X. X. Yao, Y. X. Fu, and C. B. Cai, Phys. Rev. B 54, 16211 (1996).

${ }^{22}$ G. Prando, P. Carretta, R. De Renzi, S. Sanna, A. Palenzona, M. Putti, and M. Tropeano, Phys. Rev. B 83, 174514 (2011).

${ }^{23}$ G. Prando, P. Carretta, R. De Renzi, S. Sanna, H. J. Grafe, S. Wurmehl, and B. Buchner, Phys. Rev. B 85, 144522 (2012).

${ }^{24}$ J. Ge, J. Gutierrez, J. Li, J. Yuan, H.-B. Wang, K. Yamaura, E. TakayamaMuromachi, and V. V. Moshchalkov, Phys. Rev. B 88, 144505 (2013).

${ }^{25}$ J. Li, J. Yuan, D.-M. Tang, S.-B. Zhang, M.-Y. Li, Y.-F. Guo, Y. Tsujimoto, T. Hatano, S. Arisawa, D. Golberg et al., J. Am. Chem. Soc. 134, 4068 (2012).

${ }^{26}$ J. Ge, J. Gutierrez, M. Li, J. Zhang, and V. V. Moshchalkov, Appl. Phys. Lett. 103, 052602 (2013).

${ }^{27}$ G. Blatter, M. N. Feigelman, V. B. Geschkenbein, A. I. Larkin, and V. M. Vinokur, Rev. Mod. Phys. 66, 1125 (1994).

${ }^{28}$ C. P. Bean, Phys. Rev. Lett. 8, 250 (1962).

${ }^{29}$ J. J. Qin and X. X. Yao, Phys. Rev. B 54, 7536 (1996).

${ }^{30}$ K.-H. Mller, Physica C 159, 717-726 (1989).

${ }^{31}$ M. E. McHenry, S. Simizu, H. Lessure, M. P. Maley, J. Y. Coulter, I. Tanaka, and H. Kojima, Phys. Rev. B 44, 7614 (1991).

${ }^{32}$ S. Y. Ding, J. Li, H. M. Shao, J. W. Lin, C. Ren, and X. X. Yao, Phys. Rev. B 53, 900 (1996).

${ }^{33}$ A. Houghton, R. A. Pelcovits, and A. Sudbg, Phys. Rev. B 40, 6763 (1989).

${ }^{34}$ B. Shen, P. Cheng, Z. Wang, L. Fang, C. Ren, L. Shan, and H. H. Wen, Phys. Rev. B 81, 014503 (2010).

${ }^{35}$ S. Y. Ding, G. Q. Wang, X. X. Yao, H. T. Peng, W. Y. Peng, and S. H. Zhou, Phys. Rev. B 51, 9107 (1995).

${ }^{36}$ M. P. A. Fisher, Phys. Rev. Lett. 62, 1415 (1989).

${ }^{37}$ M. Suenaga, A. K. Ghosh, Y. Xu, and D. O. Welch, Phys. Rev. Lett. 66, 1777-1780 (1991).

${ }^{38}$ X.-L. Wang, S. R. Ghorbani, S.-I. Lee, S. X. Dou, C. T. Lin, T. H. Johansen, K.-H. Muller, Z. X. Cheng, G. Peleckis, M. Shabazi et al., Phys. Rev. B 82, 024525 (2010). 
${ }^{39}$ E. Bellingeri, S. Kawale, I. Pallecchi, A. Gerbi, R. Buzio, V. Braccini, A. Palenzona, M. Putti, M. Adamo, E. Sarnelli et al., Appl. Phys. Lett. 100, 082601 (2012).

${ }^{40}$ R. Griessen, W. Hai-hu, A. J. J. van Dalen, B. Dam, J. Rector, H. G. Schnack, S. Libbrecht, E. Osquiguil, and Y. Bruynseraede, Phys. Rev. Lett. 72, 1910 (1994).

${ }^{41}$ Y. Yeshurun and A. P. Malozemoff, Phys. Rev. Lett. 60, 2202 (1988).

${ }^{42}$ M. Tinkham, Phys. Rev. Lett. 61, 1658 (1988).

${ }^{43}$ S. L. Liu, G. J. Wu, X. B. Xu, J. Wu, and H. M. Shao, Solid State Commun. 133, 615-620 (2005).

${ }^{44}$ E. Zeldov, N. M. Amer, G. Koren, A. Gupta, R. J. Gambino, and M. W. McElfresh, Phys. Rev. Lett. 62, 3093 (1989).
${ }^{45}$ J. Li, S. Y. Ding, J. S. Zhu, H. M. Shao, and Y. N. Wang, J. Appl. Phys. 77, 6398 (1995).

${ }^{46}$ S. Sengupta, D. Shi, S. Salem-Sugui, Z. Wang, P. J. McGinn, and K. DeMoranville, J. Appl. Phys. 72, 592 (1992).

${ }^{47}$ L. Baril, T. Klein, J. Marcus, and C. Escribe-Filippini, Phys. Rev. B 54, 16058 (1996).

${ }^{48}$ M. Suzuki, Phys. Rev. B 46, 14230 (1992).

${ }^{49}$ D. Lpez, L. Krusin-Elbaum, H. Safar, E. Righi, F. de la Cruz, S. Grigera, C. Feild, W. K. Kwok, L. Paulius, and G. W. Crabtree, Phys. Rev. Lett. 80, 1070 (1998).

${ }^{50}$ T. T. M. Palstra, B. Batlogg, R. B. van Dover, L. F. Schneemeyer, and J. V. Waszczak, Phys. Rev. B 41, 6621 (1990). 